\title{
Effect of Deficit Irrigation During Growth Stages on Water Use Efficiency of Carrot Under El-Ismailia Conditions
}

\author{
Ali Abdel-Aziz \\ Soil Chemistry and Physics Department - Desert Research Center
}

$\mathbf{T}$ HIS experiment was performed during the summer seasons 2015 - 2016, at a private farm in the El Kasasin area, Ismailia Governorate, Egypt, to study the effect of deficit irrigation (DI) during growth stages compared to full irrigation (FI) under surface drip (SDI) and subsurface drip (SSDI) on marketable yield (Ym), plant quality parameters, water use efficacy (WUE) and irrigation water use efficiency (IWUE) of carrot (Daucus carota L.) crop. The experimental design was a split plot design with three replicates. The obtained results indicated that, the values of quality parameters, Ym, ETa for carrot roots decreased with increasing DI during the growth stages especially (initial and development stages) except L-ascorbic acid content and total sugar content which increased with increasing DI under SDI and SSDI for both seasons. In addition; the maximum values of $\mathrm{Ym}$ for carrot roots were 8.38 and 8.56 $\mathrm{Mg}_{\text {fed }}{ }^{-1}$, respectively, under the FI $(\mathrm{I}=100, \mathrm{D}=100, \mathrm{M}=100, \mathrm{~L}=100 \%)$ and SSDI treatment. While, the minimum values of ETa were 154.00 and $157.79 \mathrm{~mm}$, respectively, under the DI ( $I=60, D=60, M=60, L=100 \%$ ) and SSDI treatment. Moreover, the values of WUE and IWUE under the DI $(I=80, D=80, M=60, L=100 \%)$ and SSDI treatment for both seasons increased significantly to reach; 50 and $28 \%$ compared with the control treatment (FI and SDI). Finally, the DI $(I=80, D=80, M=60, L=100 \%)$ and SSDI treatments save approxymately $34 \%$ of the applied irrigation water although the reduction of Ym was approxymately $4 \%$ compared with the control treatment (FI and SDI). Deficit irrigation (DI) strategy has been chosen for use in the study as it maximizes irrigation water productivity and optimizes crop yields.

Kyewords: Deficit irrigation, Actual evapotranspiration; Water use efficiency, Irrigation water use efficiency.

\section{Introduction}

Rationalization of irrigation water became a unique and necessary way to save the water used in sandy soil of Egypt. Scheduling the required irrigation water for each crop will help in minimizing the water lost during the growing season. Regulated deficit irrigation (RDI) is a strategy that applies less than the full replacement volume of water consumed by a plant, usually during a specific developmental stage. The key to the regulated deficit irrigation strategy is the timing of the deficit and the degree of the deficit applied to achieve a desired effect on the plant. Regulated deficit irrigation is water saving technology that is relatively inexpensive and easy to implement (Webber et al., 2007). When water deficit occurs during a specific crop development period, the yield response can vary depending on crop sensitivity at that growth stage. Therefore, timing the water deficit appropriately is a tool for scheduling irrigation where a limited supply of water is available. Growth of all crops is generally divided into four stages with regard to irrigation practice ; initial stage, development stage, midseason and late season stage (Allen et al., 1998). Deficit irrigation (DI) could be used to raise the crop yield to crop water consumption ratio where crops have growth stages in their development where they are tolerant to water stress (Geerts and Raes, 2009). A certain level of water stress is applied to the crops in DI strategy either during specific growth stages or throughout the growing season, without necessarily causing significant yield reduction compared with the benefits achieved by diverting saved water to irrigate other crops (Leskovar, 2010). Withholding water during the vegetative (initial) period, as opposed to the flowering (development) or fruit forming stages, has less impact on final yield. The water savings associated with regulated deficit 
irrigation are attributed to reductions in stomata conductance which occurs as a result of the plant roots encountering drying soil, and precedes any change in leaf water potential via signals (Kazemeini et al., 2009). The expectation is that any yield reduction will be insignificant compared with benefits gained through diverting the saved water to irrigated other crops (Sadgipour, 2008). A mean crop evapotranspiration of $56 \%$ of the total water requirements would be utilized during mid-season and $20 \%$ at late season stages, while the development and initial stages, respectively use $17 \%$ and $7 \%$ of the total water required by mung bean (Agugo et al., 2009). Water use efficiency may be calculated as units of dry yield per unit land area $\left(\mathrm{Y}, \mathrm{kg} \mathrm{m}^{-2}\right)$ divided by units of water consumed by the crop per unit land area (ET, $\mathrm{m}^{3} \mathrm{~m}^{-2}$, usually reported as $\mathrm{mm}$ ) to produce that yield. Another key parameter for evaluating water efficiency is the irrigation water use efficiency (IWUE, $\mathrm{kg} \mathrm{m}^{-3}$ ) which is expressed as the amount of crop yield per total irrigation water applied (Ibragimov et al., 2007). Regulated deficit irrigation is one way of maximizing water use efficiency for higher yield per unit irrigation water applied by exposing the crop to a certain level of water stress through a particular period or throughout the whole growing season (Bekele and Tilahun, 2007). Plant growth parameters measured were: fruit weight, mean fruit dimensions, number of fruit per plant. It was observed for all the crops with the exception of carrot that reducing the ETc by $20 \%$ did not significantly affect the yield components. This implies that irrigation water could be reduced when cropping these crops and the extra stretched to bring more land under production (Owusu-Sekyere and Andoh, 2011). During the experimental periods the differences in yield and its components under FI100 and FI-DI60 treatments were not significant. Reduction of water supply after mid-season stage by $40 \%$ (FI-DI60) seems to have low impact on soil salinity and yield of carrot crop as compared to full irrigation regime (Nagaz et al., 2012). We found that the highest yield of carrot crop in sandy soil was obtained with the $100 \%$ Epan treatment. The maximum WUE corresponded to $75 \%$ Epan treatment, with an applied water volume of 3864 $\mathrm{m}^{3} \mathrm{ha}^{-1}$, which corresponds to the water application level recommended for drip irrigation scheduling in carrot. The decrease applied in the water volume did not affect crop yield nor quality parameters significantly. On the other hand, the excess of soil water caused a decrease in plant density and root size. The relationship between crop yield and applied water volume obtained for the carrot crop with drip irrigation will help to improve the management of the water resources for this crop under water scarcity conditions (Quezada et al., 2011).

This study aimed to investigate the effect of deficit irrigation water during growth stages under surface and sub-surface drip irrigation on carrot root production, growth parameters quality, actual evapotranspiration (ETa), WUE and IWUE.

\section{Materials and Methods}

\section{Experiments}

Field experiments were performed in the El Kasasin area, Ismailia Governorate, Egypt $\left(30^{\circ} 29^{\circ}\right.$ $\mathrm{N}: 32^{\circ} 13^{`}$ E. $17 \mathrm{~m}$ a.s.l) during the summer seasons 2015-2016. In a split plot design with three replicates, the experimental was divided into $20 \mathrm{~m}^{2}$ plots; each bounded by $1.5 \mathrm{~m}$ wide barren to avoid horizontal infiltration. The obtained data were subjected to statistical analysis according to Snedecor and Cochran (1989) using Co-state software program. Figure 1 showed that carrot (Daucus carota L.) was irrigated by three levels of DI during growth stages (Initial ${ }_{100,80,60 \%}$ - development ${ }_{100,80,60 \%}$ - midseason $_{100,80,60 \%}$ - Late ${ }_{100 \%}$ ) by using probability tree compared with full irrigation (FI) for all growth stages (Initial ${ }_{100 \%}$ - development ${ }_{100 \%}$ - mid-season ${ }_{100 \%}$ - Late ${ }_{100 \%}$ ) under surface (SDI) and sub-surface drip irrigation (SSDI). Soil management practices were applied using doses of fertilizer as recommended by the Ministry of Agriculture and Land Reclamation.

The length (L) $\mathrm{cm}$, diameter (D) $\mathrm{cm}$, total soluble solid (TSS) \%, total carotenoid content (TCC) $\mathrm{mg} 100 \mathrm{~g}^{-1} \mathrm{FW}, \mathrm{L}$-ascorbic acid content (LAC) $\mathrm{mg} 100 \mathrm{~g}^{-1} \mathrm{FW}$ and total sugar content (TSC) $\mathrm{mg} 100 \mathrm{~g}^{-1} \mathrm{FW}$ were determined for carrot roots. WUE $\mathrm{kg} \mathrm{m}^{-3}$, IWUE $\mathrm{kg} \mathrm{m}^{-3}$ and ETa $\mathrm{mm}$, were calculated at different (DI) during growth stages under SDI and SSDI for carrot roots plots.

\section{Soil characteristics}

Soil samples were collected for some physical and chemical soil characteristics. The methodological procedures were done according to the methods described by Klute (1986) and Page et al. (1982), respectively (Tables $1 \& 2$ ).

\section{Irrigation water characteristics}

Chemical analyses of the irrigation water were measured according to the methods described by Ayers and westcot (1994) (Table 3). 


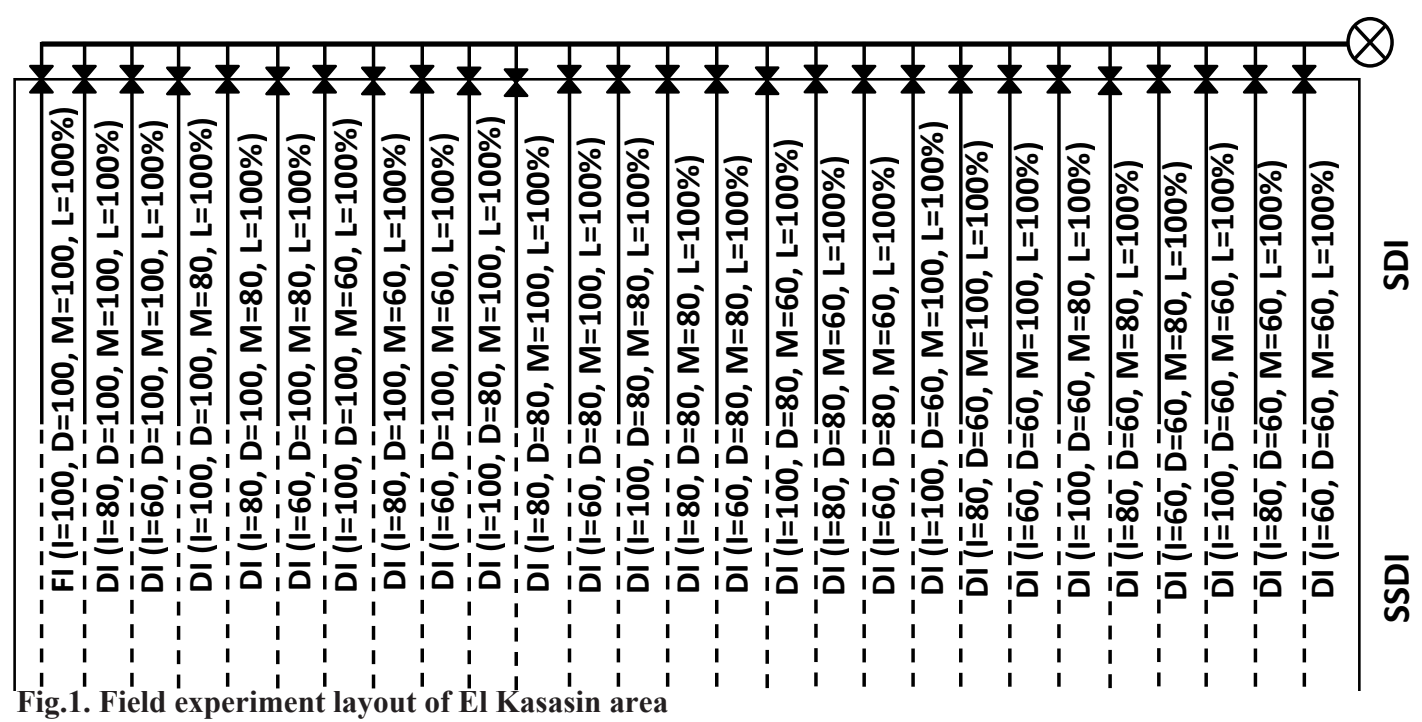

TABLE 1. Some physical characteristics of experimental soil

\begin{tabular}{|c|c|c|c|c|c|c|c|c|c|c|c|c|}
\hline \multirow{2}{*}{$\begin{array}{c}\text { Soil } \\
\text { depth } \\
(\mathrm{cm})\end{array}$} & \multicolumn{5}{|c|}{ Particle size distribution \% } & \multirow{2}{*}{$\begin{array}{l}\text { Textural } \\
\text { class }\end{array}$} & \multirow{2}{*}{$\begin{array}{c}\text { OM } \\
\%\end{array}$} & \multirow{2}{*}{$\begin{array}{c}\rho_{b} \\
\mathrm{~g} / \mathrm{cm}^{3}\end{array}$} & \multirow{2}{*}{$\begin{array}{c}\mathrm{Ks} \\
\mathrm{cm} / \mathrm{h}\end{array}$} & \multirow{2}{*}{$\begin{array}{l}\text { FC } \\
\%\end{array}$} & \multirow{2}{*}{$\begin{array}{l}\text { WP } \\
\%\end{array}$} & \multirow{2}{*}{$\begin{array}{c}\text { AW } \\
\%\end{array}$} \\
\hline & C. sand & M. sand & F. sand & Silt & Clay & & & & & & & \\
\hline $0-15$ & 8.59 & 69.15 & 10.57 & 6.76 & 4.93 & $\mathrm{~S}$ & 0.49 & 1.56 & 11.67 & 11.95 & 3.61 & 8.34 \\
\hline $15-30$ & 9.84 & 67.62 & 12.38 & 6.45 & 3.71 & $\mathrm{~S}$ & 0.47 & 1.58 & 12.14 & 11.49 & 3.53 & 7.96 \\
\hline $30-45$ & 10.17 & 65.73 & 14.45 & 6.39 & 3.26 & $\mathrm{~S}$ & 0.43 & 1.59 & 12.81 & 10.73 & 3.28 & 7.45 \\
\hline
\end{tabular}

TABLE 2. Some chemical characteristics of experimental soil

\begin{tabular}{|c|c|c|c|c|c|c|c|c|c|c|c|c|}
\hline \multirow{2}{*}{ 党 } & \multirow{2}{*}{ 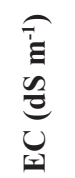 } & \multirow[b]{2}{*}{$\frac{\pi}{2}$} & \multirow{2}{*}{$\begin{array}{l}\partial^{0} \\
0^{m} \\
\bigcup_{\tilde{J}}^{m}\end{array}$} & \multirow{2}{*}{ 选 } & \multicolumn{8}{|c|}{ Soluble ions $(\mathrm{meq} / \mathrm{l})$ in the saturated soil paste extract } \\
\hline & & & & & 茫 & 戞 & 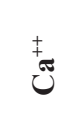 & $\sum^{+\infty}$ & $\dot{U}$ & $e^{\prime}$ & $e^{\prime m}$ & $\overbrace{}^{1}$ \\
\hline $0-15$ & 1.97 & 7.54 & 2.21 & 5.14 & 9.43 & 1.19 & 4.81 & 4.27 & 9.65 & 2.84 & - & 7.21 \\
\hline 15-30 & 2.14 & 7.42 & 2.37 & 4.34 & 9.07 & 2.31 & 5.43 & 4.59 & 10.27 & 2.79 & - & 8.34 \\
\hline $30-45$ & 2.49 & 7.37 & 2.45 & 3.95 & 8.79 & 3.94 & 6.36 & 5.81 & 12.19 & 2.63 & - & 10.08 \\
\hline
\end{tabular}

in Table 4 was calculated using the Cropwate (8) software based on Penman-Monteith equation FAO 56 method (Allen et al., 1998).

\section{Crop evapotranspiration (ETc)}

The crop evapotranspiration (ETc) shown in Table 5 was calculated using the following equation:

$$
\begin{aligned}
& \text { - } \quad \mathrm{ETc}=\mathrm{Kc}_{\mathrm{FAO}} . \text { ETo } \quad\left(\mathrm{mm} \mathrm{day}^{-1}\right) \quad \text { (Allen } \\
& \text { et al., 1998) }
\end{aligned}
$$

where: $\mathrm{Kc}_{\mathrm{FAO}}$ is the crop coefficient from FAO No.

(56). ETo is the reference crop evapotranspiration, $\mathrm{mm}$ day $^{-1}$

\section{Leaching requirement (LR)}

The leaching requirement LR was calculated using the following equation:
Where: $\mathrm{ECw}$ is the electrical conductivity of the irrigation water, $\mathrm{dS} \mathrm{m}^{-1}$.

ECe is the average electrical conductivity of the soil solution extract, $\mathrm{dS} \mathrm{m}^{-1}$.

Deficit irrigation water (DI) during growth stages

The deficit irrigation water at each growth stages based on the amounts of applied irrigation water (IR) for carrot root shown in Tables 6\&7 was calculated using the following equation:

- $\left.\quad \mathrm{IR}_{100,80,60 \%}=(\mathrm{ETc}-\mathrm{pe}) \mathrm{Kr} / \mathrm{Ea}\right)+\mathrm{LR} \quad\left(\mathrm{mmperiod}^{-1}\right)$ (Keller and Karmeli, 1974)

Where: $\mathrm{Kr}$ is correction factor for limited wetting at a carrot percentage round coverage by canopy $80 \%, \mathrm{Kr}=0.90$. (Smith, 1992).

Egypt. J. Soil Sci., 57, No. 4 (2017) 
Ea is the irrigation efficiency for surface drip (85\%) (Allen et al., 1998).

$\mathrm{Pe}$ is the effective rainfall, $0 \mathrm{~mm}$.

LR is the leaching requirements, under salinity levels of irrigation water $(0.07 \mathrm{x}$ ETc $), \mathrm{mm}$.

Convert $\mathrm{mm}$ to $\mathrm{m}^{3}=$ water per $\mathrm{mm}$ depth * Area (3.57 not 4.2 for drip irrigation)

- Actual evapotranspiration $\mathrm{ETa}=\left(\mathrm{M}_{2} \%-\mathrm{M}_{1} \%\right)$

$/ 100 . \mathrm{d}_{\mathrm{b}} . \mathrm{D}(\mathrm{mm})$ (Doorenbos and Pruitt, 1984)

Where: $\mathrm{M}_{2}$ is the moisture content after irrigation $\%$.

TABLE 3. Some chemical analysis for irrigation water
$M_{1}$ is the moisture content before irrigation $\%$. $\mathrm{d}_{\mathrm{b}}$ is the specific density of soil .

$\mathrm{D}$ is the mean depth, $\mathrm{mm}$.

- Water use efficiency WUE $=\mathrm{Ya} / \mathrm{ETa}\left(\mathrm{kgm}^{-3}\right)$ $\left(\mathrm{kg} \mathrm{m}^{-3}\right)$ (Howell et al., 2001)

Where:Ya is the actual yield of the crop, $\left(\mathrm{kg} \mathrm{fed}^{-1}\right)$.

- Irrigation water use efficiency IWUE $=\mathrm{Ya} /$ IR $\left(\mathrm{kg} \mathrm{m}^{-3}\right)$ (Michael, 1978)

Where: IR is the seasonal amounts of applied irrigation water, $\left(\mathrm{m}^{3}\right)$, Tables $(6 \& 7)$.

\begin{tabular}{|c|c|c|c|c|c|c|c|c|c|c|c|}
\hline \multirow{2}{*}{ Sample } & \multirow{2}{*}{ pH } & \multirow{2}{*}{$\begin{array}{c}E C \\
d S ~ m^{-1}\end{array}$} & \multirow{2}{*}{ SAR } & \multicolumn{4}{|c|}{ Soluble cations, meq/l } & \multicolumn{4}{|c|}{ Soluble anions, meq/l } \\
\hline & & & & $\mathbf{N a}^{+}$ & $\mathbf{K}^{+}$ & $\mathbf{C a}^{++}$ & $\mathbf{M g}^{++}$ & $\mathrm{CL}^{-}$ & $\mathrm{HCO}_{3}^{-}$ & $\mathrm{CO}_{3}=$ & $\mathrm{SO}_{4}^{=}$ \\
\hline Mean & 7.09 & 0.74 & 1.68 & 2.54 & 0.31 & 2.46 & 2.09 & 3.26 & 3.11 & - & 1.03 \\
\hline
\end{tabular}

TABLE 4. Calculation reference evapotranspiration $\left(\mathrm{mm} \mathrm{day}^{-1}\right)$ through carrot growth period

\begin{tabular}{|c|c|c|c|c|c|}
\hline Seasons & Month & August & September & October & November \\
\hline 2015 & ETo, mm day ${ }^{-1}$ & 7.03 & 5.86 & 4.51 & 2.89 \\
\hline
\end{tabular}

TABLE 5. Calculation crop evapotranspiration $\left(\mathrm{mm} \mathrm{day}^{-1}\right)$ through carrot growth period

\begin{tabular}{|c|c|c|c|c|c|c|}
\hline \multirow[t]{2}{*}{ Seasons } & $\begin{array}{r}\text { Stages } \\
\text { Period length (day) }\end{array}$ & $\begin{array}{c}\text { Initial } \\
20\end{array}$ & $\begin{array}{c}\text { Develop. } \\
30\end{array}$ & $\underset{30}{\text { Mid }}$ & $\begin{array}{c}\text { Late } \\
20\end{array}$ & $\begin{array}{c}\text { Seasonal } \\
100\end{array}$ \\
\hline & $\mathbf{K} \mathbf{c}(-)$ & 0.70 & 0.85 & 1.05 & 0.30 & -------- \\
\hline \multirow{2}{*}{2015} & $\operatorname{ETO}^{\text {FAO }}(\mathrm{mm}) 2015$ & 135.92 & 170.40 & 130.44 & 57.80 & 494.56 \\
\hline & $\mathbf{E T c}_{100 \%}(\mathrm{~mm}) 2015$ & 95.14 & 178.92 & 123.92 & 17.34 & 415.32 \\
\hline \multirow{2}{*}{2016} & ETo $(\mathrm{mm}) 2016$ & 137.72 & 173.02 & 132.45 & 58.60 & 501.79 \\
\hline & $\mathrm{ETc}_{100 \%}(\mathrm{~mm}) 2016$ & 96.40 & 181.67 & 125.83 & 17.58 & 421.48 \\
\hline
\end{tabular}

TABLE 6. Calculation applied irrigation water (IR), $\mathbf{m m}$ for carrot season 2015

\begin{tabular}{|c|c|c|c|c|c|c|c|c|}
\hline \multicolumn{4}{|c|}{ Deficit irrigation, (DI) } & \multicolumn{5}{|c|}{$\begin{array}{c}\text { Applied irrigation water, } \\
\text { Growth Stages }\end{array}$} \\
\hline 100 & $\mathbf{D}$ & $\mathbf{M}$ & 100 & Initial & Development & Mid & Late & Seasonal \\
\hline $\begin{array}{l}100 \\
80\end{array}$ & $\begin{array}{l}100 \\
100\end{array}$ & $\begin{array}{l}100 \\
100\end{array}$ & $\begin{array}{l}100 \\
100\end{array}$ & $\begin{array}{l}107.60 \\
86.08\end{array}$ & $\begin{array}{l}202.35 \\
202.35\end{array}$ & $\begin{array}{l}140.14 \\
140.14\end{array}$ & $\begin{array}{l}19.61 \\
19.61\end{array}$ & $\begin{array}{l}469.70 \\
448.18\end{array}$ \\
\hline 60 & 100 & 100 & 100 & 64.56 & 202.35 & 140.14 & 19.61 & 426.66 \\
\hline 100 & 100 & 80 & 100 & 107.60 & 202.35 & 112.11 & 19.61 & 441.67 \\
\hline 80 & 100 & 80 & 100 & 86.08 & 202.35 & 112.11 & 19.61 & 420.15 \\
\hline 60 & 100 & 80 & 100 & 64.56 & 202.35 & 112.11 & 19.61 & 398.63 \\
\hline 100 & 100 & 60 & 100 & 107.60 & 202.35 & 84.08 & 19.61 & 413.64 \\
\hline 80 & 100 & 60 & 100 & 86.08 & 202.35 & 84.08 & 19.61 & 392.12 \\
\hline 60 & 100 & 60 & 100 & 64.56 & 202.35 & 84.08 & 19.61 & 370.60 \\
\hline 100 & 80 & 100 & 100 & 107.60 & 161.88 & 140.14 & 19.61 & 429.23 \\
\hline 80 & 80 & 100 & 100 & 86.08 & 161.88 & 140.14 & 19.61 & 407.71 \\
\hline 60 & 80 & 100 & 100 & 64.56 & 161.88 & 140.14 & 19.61 & 386.19 \\
\hline 100 & 80 & 80 & 100 & 107.60 & 161.88 & 112.11 & 19.61 & 401.20 \\
\hline 80 & 80 & 80 & 100 & 86.08 & 161.88 & 112.11 & 19.61 & 379.68 \\
\hline 60 & 80 & 80 & 100 & 64.56 & 161.88 & 112.11 & 19.61 & 358.16 \\
\hline 100 & 80 & 60 & 100 & 107.60 & 161.88 & 84.08 & 19.61 & 373.17 \\
\hline 80 & 80 & 60 & 100 & 86.08 & 161.88 & 84.08 & 19.61 & 351.65 \\
\hline 60 & 80 & 60 & 100 & 64.56 & 161.88 & 84.08 & 19.61 & 330.13 \\
\hline 100 & 60 & 100 & 100 & 107.60 & 121.41 & 140.14 & 19.61 & 388.76 \\
\hline 80 & 60 & 100 & 100 & 86.08 & 121.41 & 140.14 & 19.61 & 367.24 \\
\hline 60 & 60 & 100 & 100 & 64.56 & 121.41 & 140.14 & 19.61 & 345.72 \\
\hline 100 & 60 & 80 & 100 & 107.60 & 121.41 & 112.11 & 19.61 & 360.73 \\
\hline 80 & 60 & 80 & 100 & 86.08 & 121.41 & 112.11 & 19.61 & 339.21 \\
\hline 60 & 60 & 80 & 100 & 64.56 & 121.41 & 112.11 & 19.61 & 317.69 \\
\hline 100 & 60 & 60 & 100 & 107.60 & 121.41 & 84.08 & 19.61 & 332.70 \\
\hline 80 & 60 & 60 & 100 & 86.08 & 121.41 & 84.08 & 19.61 & 311.18 \\
\hline 60 & 60 & 60 & 100 & 64.56 & 121.41 & 84.08 & 19.61 & 289.66 \\
\hline
\end{tabular}

Egypt. J. Soil Sci., 57, No. 4 (2017) 
TABLE 7. Calculation applied irrigation water (IR), $\mathbf{m m}$ for carrot season 2016

\begin{tabular}{|c|c|c|c|c|c|c|c|c|}
\hline \multicolumn{4}{|c|}{ DI } & \multicolumn{5}{|c|}{$\begin{array}{c}\text { Applied irrigation water, (mm) } \\
\text { Growth stages }\end{array}$} \\
\hline I & D & $\mathbf{M}$ & $\mathbf{L}$ & Initial & Development & Mid & Late & Seasonal \\
\hline 100 & 100 & 100 & 100 & 109.03 & 205.46 & 142.30 & 19.88 & 476.67 \\
\hline & & & 100 & 87.22 & & & 19.88 & \\
\hline 60 & 100 & 100 & 100 & 65.42 & 205.46 & 142.30 & 19.88 & 433.06 \\
\hline 100 & 100 & 80 & 100 & 109.03 & 205.46 & 113.84 & 19.88 & 448.21 \\
\hline 80 & 100 & 80 & 100 & 87.22 & 205.46 & 113.84 & 19.88 & 426.40 \\
\hline 60 & 100 & 80 & 100 & 65.42 & 205.46 & 113.84 & 19.88 & 404.60 \\
\hline 100 & 100 & 60 & 100 & 109.03 & 205.46 & 85.38 & 19.88 & 419.75 \\
\hline 80 & 100 & 60 & 100 & 87.22 & 205.46 & 85.38 & 19.88 & 397.94 \\
\hline 60 & 100 & 60 & 100 & 65.42 & 205.46 & 85.38 & 19.88 & 376.14 \\
\hline 100 & 80 & 100 & 100 & 109.03 & 164.37 & 142.30 & 19.88 & 435.58 \\
\hline 80 & 80 & 100 & 100 & 87.22 & 164.37 & 142.30 & 19.88 & 413.77 \\
\hline 60 & 80 & 100 & 100 & 65.42 & 164.37 & 142.30 & 19.88 & 391.97 \\
\hline 100 & 80 & 80 & 100 & 109.03 & 164.37 & 113.84 & 19.88 & 407.12 \\
\hline 80 & 80 & 80 & 100 & 87.22 & 164.37 & 113.84 & 19.88 & 385.31 \\
\hline 60 & 80 & 80 & 100 & 65.42 & 164.37 & 113.84 & 19.88 & 363.51 \\
\hline 100 & 80 & 60 & 100 & 109.03 & 164.37 & 85.38 & 19.88 & 378.66 \\
\hline 80 & 80 & 60 & 100 & 87.22 & 164.37 & 85.38 & 19.88 & 356.85 \\
\hline 60 & 80 & 60 & 100 & 65.42 & 164.37 & 85.38 & 19.88 & 335.05 \\
\hline 100 & 60 & 100 & 100 & 109.03 & 123.28 & 142.30 & 19.88 & 394.49 \\
\hline 80 & 60 & 100 & 100 & 87.22 & 123.28 & 142.30 & 19.88 & 372.68 \\
\hline 60 & 60 & 100 & 100 & 65.42 & 123.28 & 142.30 & 19.88 & 350.88 \\
\hline 100 & 60 & 80 & 100 & 109.03 & 123.28 & 113.84 & 19.88 & 366.03 \\
\hline 80 & 60 & 80 & 100 & 87.22 & 123.28 & 113.84 & 19.88 & 344.22 \\
\hline 60 & 60 & 80 & 100 & 65.42 & 123.28 & 113.84 & 19.88 & 322.42 \\
\hline 100 & 60 & 60 & 100 & 109.03 & 123.28 & 85.38 & 19.88 & 337.57 \\
\hline 80 & 60 & 60 & 100 & 87.22 & 123.28 & 85.38 & 19.88 & 315.76 \\
\hline 60 & 60 & 60 & 100 & 65.42 & 123.28 & 85.38 & 19.88 & 293.96 \\
\hline
\end{tabular}

\section{Results and Discussion}

Effect of DI under SDI and SSDI on quality parameters for carrot roots

Data in Tables 8, 9, 10 and 11 showed that the values of quality parameters for carrot roots (length (L) $\mathrm{cm}$, diameter (D) $\mathrm{cm}$, total soluble solid (TSS) \%, total carotenoid content (TCC) mg100 $\mathrm{g}^{-1} \mathrm{FW}$ decreased with increasing deficit irrigation DI during growth stages.

The maximum values of carrot roots L, D, TSS and TCC for seasons 2015 - 2016 recorded $(17.02 \mathrm{~cm}, 3.92 \mathrm{~cm}, 12.27 \%$ and $18.51 \mathrm{mg} 100$ $\left.\mathrm{g}^{-1} \mathrm{fw}\right)$ and $(17.23 \mathrm{~cm}, 3.97 \mathrm{~cm}, 12.43 \%$ and 18.73 $\left.\mathrm{mg} 100 \mathrm{~g}^{-1} \mathrm{fw}\right)$, respectively. Whereas LAC and TSC recorded (6.79 and $\left.4.81 \mathrm{mg} 100 \mathrm{~g}^{-1} \mathrm{fw}\right)$ and (6.87 and $4.87 \mathrm{mg} 100 \mathrm{~g}^{-1} \mathrm{fw}$ ) for both seasons respectively, under full irrigation (FI) $(\mathrm{I}=100$, $\mathrm{D}=100, \mathrm{M}=100, \mathrm{~L}=100 \%$ ) and SSDI treatment.

While, the minimum values for both seasons recorded $(7.39 \mathrm{~cm}, 0.38 \mathrm{~cm}, 4.21 \%$ and 14.60 mg $\left.100 \mathrm{~g}^{-1} \mathrm{fw}\right)$ and $(7.47 \mathrm{~cm}, 0.39 \mathrm{~cm}, 4.26 \%$ and $\left.14.76 \mathrm{mg} 100 \mathrm{~g}^{-1} \mathrm{fw}\right)$, respectively. Whereas LAC and TSC recorded (7.43 and $5.45 \mathrm{mg} 100$

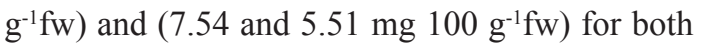
seasons, respectively, under the DI $(I=60, D=60$, $\mathrm{M}=60, \mathrm{~L}=100 \%$ ) and SDI treatment.
The values of $\mathrm{L}, \mathrm{D}, \mathrm{TSS}, \mathrm{TCC}, \mathrm{LAC}$ and TSC under full irrigation and SSDI treatment for both seasons were recorded increased significantly by about $(14,13,12,9,8$ and $11 \%)$ respectively, compared to that under control treatment. These results are similar to these reported by (Nagaz et al., 2012).

\section{Effect of DI under SDI and SSDI on marketable yield for carrot roots}

Data in Tables $12 \& 13$ show that the values of marketable yield $(\mathrm{Ym})$ for carrot roots decreased with increasing DI during growth stages under SDI and SSDI for both seasons 2015 - 2016. The maximum values of Ym were 8.38 and $8.56 \mathrm{Mg}$ fed $^{-}$ ${ }^{1}$ for both seasons, respectively, under FI ( $\mathrm{I}=100$, $\mathrm{D}=100, \mathrm{M}=100, \mathrm{~L}=100 \%$ ) and SSDI treatment. While, the minimum values of Ym were 2.39 and $2.46 \mathrm{Mg} \mathrm{fed}^{-1}$ for both seasons, respectively, under DI ( $\mathrm{I}=60, \mathrm{D}=60, \mathrm{M}=60, \mathrm{~L}=100 \%)$ and SDI treatment. Meanwhile, the values of carrot roots Ym under FI and SSDI treatment for both seasons, respectively, increased significantly by approximately $14 \%$ compared the control treatment. These results may be attributed to that the initial and development growth stages of carrot roots were very sensitive for DI.These results are cosistent with the finding of Quezada et al. (2011). 
TABLE 8. Effect of deficit irrigation for carrot root growth stages on L, D and TSS under SDI and SSDI for season 2015

\begin{tabular}{|c|c|c|c|c|c|c|c|c|c|}
\hline DI & $\underset{(\mathrm{cm})}{\mathrm{L}}$ & $\begin{array}{c}D \\
(\mathbf{c m})\end{array}$ & $\begin{array}{c}\text { TSS } \\
(\%)\end{array}$ & & & & & & \\
\hline \multicolumn{4}{|c|}{ Growth stageS } & \multicolumn{6}{|c|}{ Irrigation systems (IS) } \\
\hline I & D & $\mathbf{M}$ & $\mathbf{L}$ & SDI & SSDI & SDI & SSDI & SDI & SSDI \\
\hline 100 & 100 & 100 & 100 & 14.98 & 17.02 & 3.48 & 3.92 & 10.94 & 12.27 \\
\hline 80 & 100 & 100 & 100 & 14.76 & 16.67 & 3.35 & 3.77 & 10.61 & 11.93 \\
\hline 60 & 100 & 100 & 100 & 13.54 & 15.25 & 2.84 & 3.20 & 9.12 & 10.19 \\
\hline 100 & 100 & 80 & 100 & 14.87 & 16.79 & 3.41 & 3.83 & 10.76 & 12.07 \\
\hline 80 & 100 & 80 & 100 & 14.35 & 16.07 & 3.27 & 3.68 & 10.38 & 11.58 \\
\hline 60 & 100 & 80 & 100 & 14.02 & 15.65 & 3.14 & 3.50 & 9.94 & 11.05 \\
\hline 100 & 100 & 60 & 100 & 14.14 & 15.93 & 3.09 & 3.46 & 10.09 & 11.23 \\
\hline 80 & 100 & 60 & 100 & 13.97 & 15.52 & 3.04 & 3.39 & 9.87 & 10.97 \\
\hline 60 & 100 & 60 & 100 & 12.85 & 14.19 & 2.36 & 2.61 & 10.52 & 11.65 \\
\hline 100 & 80 & 100 & 100 & 14.63 & 16.27 & 3.30 & 3.67 & 10.39 & 11.51 \\
\hline 80 & 80 & 100 & 100 & 14.49 & 16.04 & 3.22 & 3.55 & 10.25 & 11.25 \\
\hline 60 & 80 & 100 & 100 & 13.82 & 15.15 & 2.80 & 3.08 & 9.08 & 9.92 \\
\hline 100 & 80 & 80 & 100 & 14.76 & 16.06 & 3.26 & 3.54 & 10.53 & 11.54 \\
\hline 80 & 80 & 80 & 100 & 14.54 & 15.64 & 3.14 & 3.41 & 10.11 & 11.06 \\
\hline 60 & 80 & 80 & 100 & 13.37 & 14.32 & 2.76 & 2.99 & 8.56 & 9.31 \\
\hline 100 & 80 & 60 & 100 & 13.71 & 14.81 & 2.90 & 3.13 & 8.84 & 9.63 \\
\hline 80 & 80 & 60 & 100 & 13.48 & 14.39 & 2.82 & 3.04 & 8.69 & 9.44 \\
\hline 60 & 80 & 60 & 100 & 13.00 & 13.84 & 2.58 & 2.80 & 8.02 & 8.71 \\
\hline 100 & 60 & 100 & 100 & 11.23 & 12.07 & 1.33 & 1.44 & 6.61 & 7.18 \\
\hline 80 & 60 & 100 & 100 & 9.98 & 10.69 & 1.11 & 1.20 & 5.45 & 5.91 \\
\hline 60 & 60 & 100 & 100 & 9.16 & 9.72 & 0.78 & 0.85 & 5.21 & 5.59 \\
\hline 100 & 60 & 80 & 100 & 10.94 & 11.68 & 1.26 & 1.37 & 6.38 & 6.85 \\
\hline 80 & 60 & 80 & 100 & 9.79 & 10.40 & 0.92 & 1.02 & 5.32 & 5.73 \\
\hline 60 & 60 & 80 & 100 & 8.67 & 9.13 & 0.64 & 0.71 & 4.92 & 5.28 \\
\hline 100 & 60 & 60 & 100 & 9.90 & 10.49 & 0.97 & 1.05 & 5.34 & 5.76 \\
\hline 80 & 60 & 60 & 100 & 8.84 & 9.30 & 0.71 & 0.78 & 5.07 & 5.45 \\
\hline 60 & 60 & 60 & 100 & 7.39 & 7.73 & 0.38 & 0.42 & 4.21 & 4.52 \\
\hline \multicolumn{4}{|c|}{ IS } & \multicolumn{2}{|c|}{0.02} & \multicolumn{2}{|c|}{0.01} & \multicolumn{2}{|c|}{0.02} \\
\hline \multirow{2}{*}{\multicolumn{2}{|c|}{ LSD 0.05}} & \multicolumn{2}{|c|}{ DI } & \multicolumn{2}{|c|}{0.05} & \multicolumn{2}{|c|}{0.01} & \multicolumn{2}{|c|}{0.04} \\
\hline & & \multicolumn{2}{|c|}{ IS X DI } & \multicolumn{2}{|c|}{0.07} & \multicolumn{2}{|c|}{0.03} & \multicolumn{2}{|c|}{0.05} \\
\hline
\end{tabular}


TABLE 9. Effect of deficit irrigation for carrot root growth stages on LAC, TSC and TCC under SDI and SSDI for season 2015

\begin{tabular}{|c|c|c|c|c|c|c|c|c|c|}
\hline DI & $\begin{array}{c}\text { TCC } \\
\left(\mathrm{mg} \mathrm{100} \mathrm{g}^{-1}\right. \\
\text { FW) }\end{array}$ & $\begin{array}{c}\text { LAC } \\
(\mathrm{mg} \mathrm{100} \\
\left.\mathrm{g}^{-1} \mathrm{FW}\right)\end{array}$ & $\begin{array}{c}\text { TSC } \\
(\mathrm{mg} 100 \\
\left.\mathrm{g}^{-1} \mathrm{FW}\right)\end{array}$ & & & & & & \\
\hline \multicolumn{4}{|c|}{ Growth stageS } & \multicolumn{6}{|c|}{ Irrigation systems (IS) } \\
\hline $\mathbf{I}$ & D & $\mathbf{M}$ & $\mathbf{L}$ & SDI & SSDI & SDI & SSDI & SDI & SSDI \\
\hline 100 & 100 & 100 & 100 & 16.94 & 18.51 & 6.27 & 6.79 & 4.34 & 4.81 \\
\hline 80 & 100 & 100 & 100 & 16.72 & 18.27 & 6.35 & 6.87 & 4.42 & 4.89 \\
\hline 60 & 100 & 100 & 100 & 16.47 & 18.03 & 6.43 & 6.95 & 4.56 & 5.04 \\
\hline 100 & 100 & 80 & 100 & 16.69 & 18.29 & 6.34 & 6.90 & 4.40 & 4.87 \\
\hline 80 & 100 & 80 & 100 & 16.51 & 18.05 & 6.41 & 7.02 & 4.48 & 5.06 \\
\hline 60 & 100 & 80 & 100 & 16.25 & 17.81 & 6.49 & 7.11 & 4.62 & 5.12 \\
\hline 100 & 100 & 60 & 100 & 16.43 & 18.07 & 6.43 & 7.02 & 4.49 & 4.95 \\
\hline 80 & 100 & 60 & 100 & 16.28 & 17.83 & 6.51 & 7.14 & 4.56 & 5.02 \\
\hline 60 & 100 & 60 & 100 & 16.06 & 17.65 & 6.65 & 7.26 & 4.68 & 5.17 \\
\hline 100 & 80 & 100 & 100 & 16.20 & 17.89 & 6.54 & 7.09 & 4.57 & 5.09 \\
\hline 80 & 80 & 100 & 100 & 16.02 & 17.67 & 6.63 & 7.16 & 4.63 & 5.15 \\
\hline 60 & 80 & 100 & 100 & 15.89 & 17.41 & 6.71 & 7.24 & 4.75 & 5.23 \\
\hline 100 & 80 & 80 & 100 & 16.04 & 17.65 & 6.67 & 7.21 & 4.65 & 5.17 \\
\hline 80 & 80 & 80 & 100 & 15.86 & 17.43 & 6.74 & 7.32 & 4.71 & 5.24 \\
\hline 60 & 80 & 80 & 100 & 15.62 & 17.19 & 6.86 & 7.45 & 4.83 & 5.31 \\
\hline 100 & 80 & 60 & 100 & 15.84 & 17.32 & 6.79 & 7.30 & 4.73 & 5.26 \\
\hline 80 & 80 & 60 & 100 & 15.68 & 17.10 & 6.85 & 7.37 & 4.79 & 5.38 \\
\hline 60 & 80 & 60 & 100 & 15.46 & 16.94 & 6.97 & 7.49 & 4.87 & 5.53 \\
\hline 100 & 60 & 100 & 100 & 15.60 & 17.08 & 6.91 & 7.43 & 4.85 & 5.40 \\
\hline 80 & 60 & 100 & 100 & 15.43 & 16.86 & 6.98 & 7.51 & 4.97 & 5.56 \\
\hline 60 & 60 & 100 & 100 & 15.25 & 16.62 & 7.11 & 7.65 & 5.14 & 5.71 \\
\hline 100 & 60 & 80 & 100 & 15.39 & 16.84 & 7.05 & 7.57 & 5.00 & 5.58 \\
\hline 80 & 60 & 80 & 100 & 15.17 & 16.60 & 7.13 & 7.69 & 5.15 & 5.73 \\
\hline 60 & 60 & 80 & 100 & 14.91 & 16.38 & 7.27 & 7.83 & 5.29 & 5.87 \\
\hline 100 & 60 & 60 & 100 & 15.13 & 16.65 & 7.19 & 7.71 & 5.17 & 5.75 \\
\hline 80 & 60 & 60 & 100 & 14.85 & 16.43 & 7.31 & 7.83 & 5.32 & 5.89 \\
\hline 60 & 60 & 60 & 100 & 14.60 & 16.21 & 7.43 & 7.97 & 5.45 & 6.11 \\
\hline & & \multicolumn{2}{|c|}{ IS } & \multicolumn{2}{|c|}{0.03} & \multicolumn{2}{|c|}{0.02} & \multicolumn{2}{|c|}{0.01} \\
\hline \multicolumn{2}{|c|}{ LSD 0.05} & \multicolumn{2}{|c|}{ DI } & \multicolumn{2}{|c|}{0.06} & \multicolumn{2}{|c|}{0.03} & \multicolumn{2}{|c|}{0.02} \\
\hline & & \multicolumn{2}{|c|}{ IS X DI } & \multicolumn{2}{|c|}{0.09} & \multicolumn{2}{|c|}{0.05} & \multicolumn{2}{|c|}{0.03} \\
\hline
\end{tabular}


TABLE 10. Effect of deficit irrigation for carrot root growth stages on L, SD and TSS under SDI and SSDI for season 2016

\begin{tabular}{|c|c|c|c|c|c|c|c|c|c|}
\hline DI & $\begin{array}{c}\mathbf{L} \\
(\mathbf{c m})\end{array}$ & $\begin{array}{l}\text { SD } \\
(\mathbf{c m})\end{array}$ & $\begin{array}{c}\text { TSS } \\
(\%)\end{array}$ & & & & & & \\
\hline \multicolumn{4}{|c|}{ Growth stages } & \multicolumn{6}{|c|}{ Irrigation systems (IS) } \\
\hline I & $\mathrm{D}$ & M & $\mathrm{L}$ & SDI & SSDI & SDI & SSDI & SDI & SSDI \\
\hline 100 & 100 & 100 & 100 & 15.19 & 17.23 & 3.52 & 3.97 & 11.07 & 12.43 \\
\hline 80 & 100 & 100 & 100 & 14.95 & 16.86 & 3.39 & 3.81 & 10.75 & 12.07 \\
\hline 60 & 100 & 100 & 100 & 13.71 & 15.42 & 2.87 & 3.23 & 9.23 & 10.30 \\
\hline 100 & 100 & 80 & 100 & 15.03 & 16.99 & 3.45 & 3.87 & 10.90 & 12.21 \\
\hline 80 & 100 & 80 & 100 & 14.50 & 16.28 & 3.31 & 3.72 & 10.49 & 11.73 \\
\hline 60 & 100 & 80 & 100 & 14.17 & 15.86 & 3.18 & 3.54 & 10.06 & 11.18 \\
\hline 100 & 100 & 60 & 100 & 14.34 & 16.12 & 3.13 & 3.50 & 10.21 & 11.36 \\
\hline 80 & 100 & 60 & 100 & 14.12 & 15.70 & 3.07 & 3.42 & 9.98 & 11.09 \\
\hline 60 & 100 & 60 & 100 & 12.96 & 14.35 & 2.39 & 2.64 & 10.65 & 11.80 \\
\hline 100 & 80 & 100 & 100 & 14.81 & 16.48 & 3.34 & 3.71 & 10.53 & 11.65 \\
\hline 80 & 80 & 100 & 100 & 14.64 & 16.25 & 3.26 & 3.59 & 10.37 & 11.38 \\
\hline 60 & 80 & 100 & 100 & 14.00 & 15.32 & 2.83 & 3.11 & 9.20 & 10.03 \\
\hline 100 & 80 & 80 & 100 & 14.93 & 16.23 & 3.30 & 3.58 & 10.65 & 11.67 \\
\hline 80 & 80 & 80 & 100 & 14.68 & 15.85 & 3.18 & 3.45 & 10.23 & 11.19 \\
\hline 60 & 80 & 80 & 100 & 13.51 & 14.51 & 2.79 & 3.02 & 8.67 & 9.42 \\
\hline 100 & 80 & 60 & 100 & 13.89 & 14.99 & 2.93 & 3.16 & 8.95 & 9.76 \\
\hline 80 & 80 & 60 & 100 & 13.63 & 14.58 & 2.85 & 3.07 & 8.78 & 9.54 \\
\hline 60 & 80 & 60 & 100 & 13.17 & 14.01 & 2.61 & 2.83 & 8.12 & 8.81 \\
\hline 100 & 60 & 100 & 100 & 11.35 & 12.20 & 1.34 & 1.45 & 6.69 & 7.27 \\
\hline 80 & 60 & 100 & 100 & 10.13 & 10.82 & 1.12 & 1.21 & 5.51 & 5.98 \\
\hline 60 & 60 & 100 & 100 & 9.27 & 9.85 & 0.79 & 0.86 & 5.27 & 5.65 \\
\hline 100 & 60 & 80 & 100 & 11.09 & 11.83 & 1.27 & 1.38 & 6.45 & 6.93 \\
\hline 80 & 60 & 80 & 100 & 9.91 & 10.51 & 0.93 & 1.03 & 5.38 & 5.80 \\
\hline 60 & 60 & 80 & 100 & 8.78 & 9.25 & 0.65 & 0.72 & 4.97 & 5.35 \\
\hline 100 & 60 & 60 & 100 & 10.02 & 10.61 & 0.98 & 1.06 & 5.40 & 5.83 \\
\hline 80 & 60 & 60 & 100 & 8.95 & 9.41 & 0.72 & 0.79 & 5.12 & 5.51 \\
\hline \multirow[t]{2}{*}{60} & 60 & 60 & 100 & 7.47 & 7.82 & 0.39 & 0.43 & 4.26 & 4.58 \\
\hline & & \multicolumn{2}{|c|}{ IS } & \multicolumn{2}{|c|}{0.03} & \multicolumn{2}{|c|}{0.01} & \multicolumn{2}{|c|}{0.03} \\
\hline \multicolumn{2}{|c|}{ LSD 0.05} & \multicolumn{2}{|c|}{ DI } & \multicolumn{2}{|c|}{0.05} & \multicolumn{2}{|c|}{0.02} & \multicolumn{2}{|c|}{0.05} \\
\hline & & \multicolumn{2}{|c|}{ IS X DI } & \multicolumn{2}{|c|}{0.08} & \multicolumn{2}{|c|}{0.04} & \multicolumn{2}{|c|}{0.07} \\
\hline
\end{tabular}


TABLE 11. Effect of defect irrigation for carrot root growth stages on LA, TS and TC under SDI and SSDI for season 2016

\begin{tabular}{|c|c|c|c|c|c|c|c|c|c|}
\hline DI & $\begin{array}{c}\text { TC } \\
(\mathrm{mg} 100 \\
\left.\mathrm{g}^{-1} \mathrm{FW}\right)\end{array}$ & $\begin{array}{c}\text { LA } \\
\left(\mathrm{mg} 100 \mathrm{~g}^{-1}\right. \\
\text { FW) }\end{array}$ & $\begin{array}{c}\text { TS } \\
\left(\mathrm{mg} 100 \mathrm{~g}^{-1}\right. \\
\text { FW) }\end{array}$ & & & & & & \\
\hline \multicolumn{4}{|c|}{ Growth stageS } & \multicolumn{6}{|c|}{ Irrigation systems (IS) } \\
\hline I & D & $\mathbf{M}$ & $\mathbf{L}$ & SDI & SSDI & SDI & SSDI & SDI & SSDI \\
\hline 100 & 100 & 100 & 100 & 17.16 & 18.73 & 6.35 & 6.87 & 4.39 & 4.87 \\
\hline 80 & 100 & 100 & 100 & 16.94 & 18.48 & 6.43 & 6.95 & 4.47 & 4.95 \\
\hline 60 & 100 & 100 & 100 & 16.68 & 18.25 & 6.51 & 7.03 & 4.61 & 5.10 \\
\hline 100 & 100 & 80 & 100 & 16.89 & 18.51 & 6.42 & 6.98 & 4.45 & 4.93 \\
\hline 80 & 100 & 80 & 100 & 16.67 & 18.29 & 6.49 & 7.10 & 4.53 & 5.12 \\
\hline 60 & 100 & 80 & 100 & 16.42 & 18.07 & 6.57 & 7.19 & 4.67 & 5.18 \\
\hline 100 & 100 & 60 & 100 & 16.65 & 18.30 & 6.51 & 7.12 & 4.54 & 5.02 \\
\hline 80 & 100 & 60 & 100 & 16.48 & 18.04 & 6.6 & 7.24 & 4.61 & 5.09 \\
\hline 60 & 100 & 60 & 100 & 16.20 & 17.85 & 6.74 & 7.36 & 4.73 & 5.24 \\
\hline 100 & 80 & 100 & 100 & 16.42 & 18.13 & 6.62 & 7.18 & 4.62 & 5.16 \\
\hline 80 & 80 & 100 & 100 & 16.17 & 17.91 & 6.71 & 7.25 & 4.68 & 5.21 \\
\hline 60 & 80 & 100 & 100 & 16.09 & 17.57 & 6.79 & 7.33 & 4.80 & 5.29 \\
\hline 100 & 80 & 80 & 100 & 16.23 & 17.85 & 6.76 & 7.31 & 4.71 & 5.24 \\
\hline 80 & 80 & 80 & 100 & 16.01 & 17.68 & 6.83 & 7.42 & 4.77 & 5.31 \\
\hline 60 & 80 & 80 & 100 & 15.75 & 17.43 & 6.95 & 7.56 & 4.89 & 5.37 \\
\hline 100 & 80 & 60 & 100 & 16.04 & 17.51 & 6.87 & 7.39 & 4.78 & 5.33 \\
\hline 80 & 80 & 60 & 100 & 15.86 & 17.35 & 6.95 & 7.46 & 4.84 & 5.45 \\
\hline 60 & 80 & 60 & 100 & 15.68 & 17.17 & 7.07 & 7.58 & 4.92 & 5.58 \\
\hline 100 & 60 & 100 & 100 & 15.72 & 17.29 & 7.01 & 7.53 & 4.91 & 5.47 \\
\hline 80 & 60 & 100 & 100 & 15.64 & 17.11 & 7.08 & 7.61 & 5.03 & 5.64 \\
\hline 60 & 60 & 100 & 100 & 15.46 & 16.85 & 7.21 & 7.75 & 5.20 & 5.79 \\
\hline 100 & 60 & 80 & 100 & 15.58 & 17.07 & 7.15 & 7.67 & 5.06 & 5.64 \\
\hline 80 & 60 & 80 & 100 & 15.36 & 16.79 & 7.23 & 7.79 & 5.21 & 5.79 \\
\hline 60 & 60 & 80 & 100 & 15.13 & 16.61 & 7.37 & 7.93 & 5.35 & 5.93 \\
\hline 100 & 60 & 60 & 100 & 15.31 & 16.83 & 7.29 & 7.82 & 5.23 & 5.81 \\
\hline 80 & 60 & 60 & 100 & 15.04 & 16.57 & 7.41 & 7.94 & 5.38 & 5.95 \\
\hline 60 & 60 & 60 & 100 & 14.76 & 16.39 & 7.54 & 8.08 & 5.51 & 6.18 \\
\hline \multicolumn{4}{|c|}{ IS } & \multicolumn{2}{|c|}{0.03} & \multicolumn{2}{|c|}{0.02} & \multicolumn{2}{|c|}{0.02} \\
\hline \multirow{2}{*}{\multicolumn{2}{|c|}{ LSD 0.05}} & \multicolumn{2}{|c|}{ DI } & \multicolumn{2}{|c|}{0.07} & \multicolumn{2}{|c|}{0.04} & \multicolumn{2}{|c|}{0.02} \\
\hline & & \multicolumn{2}{|c|}{ IS X DI } & \multicolumn{2}{|c|}{0.09} & \multicolumn{2}{|c|}{0.06} & \multicolumn{2}{|c|}{0.04} \\
\hline
\end{tabular}


Effect of DI under SDI and SSDI on seasonal actual evapotranspiration

Data in Tables 12\&13 concluded that the values of seasonal ETa for carrot roots decreased with increasing DI during growth stages under SDI and SSDI for both seasons 2015-2016. The minimum values of ETa were 154.00 and $157.79 \mathrm{~mm}$ for both seasons, respectively, under

TABLE 12. Effect of deficit irrigation for carrot root growth stages on Ym, ETa, WUE and IWUE under SDI and SSDI for season 2015

\begin{tabular}{|c|c|c|c|c|c|c|c|c|c|c|c|}
\hline DI & $\begin{array}{c}\text { Ym } \\
\left(\mathrm{Mg}^{-1}\right. \\
\left.\text { fed }^{-1}\right)\end{array}$ & $\begin{array}{c}\text { ETa } \\
(\mathrm{mm} \\
\left.\text { Season }^{-1}\right)\end{array}$ & $\begin{array}{c}\text { WUE } \\
\left(\mathrm{Kg} \mathrm{m}^{-3}\right)\end{array}$ & $\begin{array}{c}\text { IWUE } \\
(\mathrm{Kg} \\
\left.\mathrm{m}^{-3}\right)\end{array}$ & & & & & & & \\
\hline \multicolumn{4}{|c|}{ Growth stages } & \multicolumn{8}{|c|}{ Irrigation systems (IS) } \\
\hline I & D & M & $\mathbf{L}$ & SDI & SSDI & SDI & SSDI & SDI & SSDI & SDI & SSDI \\
\hline 100 & 100 & 100 & 100 & 7.34 & 8.38 & 311.95 & 284.38 & 6.59 & 8.25 & 4.38 & 5.00 \\
\hline 80 & 100 & 100 & 100 & 7.12 & 8.05 & 278.31 & 255.19 & 7.17 & 8.84 & 4.45 & 5.03 \\
\hline 60 & 100 & 100 & 100 & 6.29 & 7.12 & 254.56 & 234.56 & 6.92 & 8.50 & 4.13 & 4.67 \\
\hline 100 & 100 & 80 & 100 & 7.25 & 8.19 & 275.42 & 250.61 & 7.37 & 9.15 & 4.60 & 5.19 \\
\hline 80 & 100 & 80 & 100 & 6.97 & 7.82 & 253.24 & 229.63 & 7.71 & 9.54 & 4.65 & 5.21 \\
\hline 60 & 100 & 80 & 100 & 6.72 & 7.48 & 237.73 & 216.49 & 7.92 & 9.68 & 4.72 & 5.26 \\
\hline 100 & 100 & 60 & 100 & 6.80 & 7.53 & 243.08 & 224.52 & 7.84 & 9.39 & 4.60 & 5.10 \\
\hline 80 & 100 & 60 & 100 & 6.69 & 7.49 & 236.63 & 220.71 & 7.92 & 9.51 & 4.78 & 5.35 \\
\hline 60 & 100 & 60 & 100 & 5.44 & 6.04 & 200.53 & 187.28 & 7.60 & 9.03 & 4.11 & 4.57 \\
\hline 100 & 80 & 100 & 100 & 7.02 & 7.87 & 293.45 & 269.53 & 6.70 & 8.18 & 4.58 & 5.14 \\
\hline 80 & 80 & 100 & 100 & 6.90 & 7.68 & 252.13 & 230.99 & 7.67 & 9.31 & 4.74 & 5.28 \\
\hline 60 & 80 & 100 & 100 & 6.30 & 7.06 & 239.08 & 218.80 & 7.38 & 9.04 & 4.57 & 5.12 \\
\hline 100 & 80 & 80 & 100 & 7.05 & 7.61 & 256.86 & 238.58 & 7.69 & 8.93 & 4.92 & 5.31 \\
\hline 80 & 80 & 80 & 100 & 6.82 & 7.29 & 225.01 & 209.34 & 8.49 & 9.75 & 5.03 & 5.38 \\
\hline 60 & 80 & 80 & 100 & 6.01 & 6.47 & 213.53 & 197.51 & 7.88 & 9.18 & 4.70 & 5.06 \\
\hline 100 & 80 & 60 & 100 & 6.18 & 7.00 & 228.15 & 211.75 & 7.59 & 9.26 & 4.64 & 5.25 \\
\hline 80 & 80 & 60 & 100 & 6.05 & 7.04 & 214.09 & 199.99 & 7.92 & 9.86 & 4.82 & 5.61 \\
\hline 60 & 80 & 60 & 100 & 5.76 & 6.12 & 202.26 & 187.28 & 7.98 & 9.15 & 4.89 & 5.19 \\
\hline 100 & 60 & 100 & 100 & 4.31 & 4.79 & 250.62 & 230.56 & 4.82 & 5.82 & 3.11 & 3.45 \\
\hline 80 & 60 & 100 & 100 & 3.82 & 4.28 & 233.75 & 216.68 & 4.58 & 5.53 & 2.91 & 3.26 \\
\hline 60 & 60 & 100 & 100 & 3.32 & 3.61 & 212.07 & 193.35 & 4.39 & 5.23 & 2.69 & 2.92 \\
\hline 100 & 60 & 80 & 100 & 4.14 & 4.53 & 226.84 & 210.42 & 5.11 & 6.03 & 3.21 & 3.52 \\
\hline 80 & 60 & 80 & 100 & 3.66 & 3.91 & 207.46 & 189.36 & 4.94 & 5.78 & 3.02 & 3.23 \\
\hline 60 & 60 & 80 & 100 & 3.04 & 3.28 & 184.12 & 168.23 & 4.62 & 5.46 & 2.68 & 2.89 \\
\hline 100 & 60 & 60 & 100 & 3.69 & 3.97 & 200.38 & 184.58 & 5.16 & 6.02 & 3.11 & 3.34 \\
\hline 80 & 60 & 60 & 100 & 3.17 & 3.34 & 181.26 & 167.71 & 4.90 & 5.58 & 2.85 & 3.01 \\
\hline 60 & 60 & 60 & 100 & 2.39 & 2.53 & 168.89 & 154.00 & 3.96 & 4.60 & 2.31 & 2.45 \\
\hline \multicolumn{4}{|c|}{ IS } & \multicolumn{2}{|c|}{0.01} & \multicolumn{2}{|c|}{0.96} & \multicolumn{2}{|c|}{0.04} & \multicolumn{2}{|c|}{0.01} \\
\hline \multirow{2}{*}{\multicolumn{2}{|c|}{ LSD 0.05}} & \multicolumn{2}{|c|}{ DI } & \multicolumn{2}{|c|}{0.06} & \multicolumn{2}{|c|}{5.69} & \multicolumn{2}{|c|}{0.20} & \multicolumn{2}{|c|}{0.05} \\
\hline & & \multicolumn{2}{|c|}{ IS X DI } & \multicolumn{2}{|c|}{0.07} & \multicolumn{2}{|c|}{5.01} & \multicolumn{2}{|c|}{0.20} & \multicolumn{2}{|c|}{0.04} \\
\hline
\end{tabular}

DI $(\mathrm{I}=60, \mathrm{D}=60, \mathrm{M}=60, \mathrm{~L}=100 \%)$ and SSDI treatment. While, the maximum values of ETa were 311.95 and $317.25 \mathrm{~mm}$ for both seasons, respectively, under FI $(\mathrm{I}=100, \mathrm{D}=100, \mathrm{M}=100$, $\mathrm{L}=100 \%$ ) and SDI treatment. These results are consisten with Webber et al. (2007) and Geerts and Raes (2009). 
TABLE 13. Effect of defect irrigation for carrot root growth stages on Ym, ETa, WUE and IWUE under SDI and SSDI for season 2016

\begin{tabular}{|c|c|c|c|c|c|c|c|c|c|c|c|}
\hline DI & $\begin{array}{l}\text { Ym } \\
(\mathrm{Mg} \\
\left.\text { fed }^{-1}\right)\end{array}$ & $\begin{array}{c}\text { ETa } \\
(\mathrm{mm} \\
\left.\text { Season }^{-1}\right)\end{array}$ & $\begin{array}{c}\text { WUE } \\
\left(\mathrm{Kg} \mathrm{m}^{-3}\right)\end{array}$ & $\begin{array}{l}\text { IWUE } \\
\left(\mathrm{Kg} \mathrm{m}^{-3}\right.\end{array}$ & & & & & & & \\
\hline & Gr & vth stages & & & & Irrig & ion syste & is (IS) & & & \\
\hline I & D & $\mathbf{M}$ & $\mathbf{L}$ & SDI & SSDI & SDI & SSDI & SDI & SSDI & SDI & SSDI \\
\hline 100 & 100 & 100 & 100 & 7.51 & 8.56 & 317.25 & 289.65 & 6.63 & 8.28 & 4.41 & 5.03 \\
\hline 80 & 100 & 100 & 100 & 7.27 & 8.23 & 283.57 & 260.03 & 7.18 & 8.87 & 4.48 & 5.07 \\
\hline 60 & 100 & 100 & 100 & 6.45 & 7.29 & 260.06 & 239.26 & 6.95 & 8.53 & 4.17 & 4.72 \\
\hline 100 & 100 & 80 & 100 & 7.39 & 8.35 & 279.34 & 254.48 & 7.41 & 9.19 & 4.62 & 5.22 \\
\hline 80 & 100 & 80 & 100 & 7.12 & 7.97 & 257.71 & 233.74 & 7.74 & 9.55 & 4.68 & 5.24 \\
\hline 60 & 100 & 80 & 100 & 6.86 & 7.61 & 241.49 & 219.51 & 7.96 & 9.71 & 4.75 & 5.27 \\
\hline 100 & 100 & 60 & 100 & 6.94 & 7.70 & 246.93 & 229.40 & 7.87 & 9.40 & 4.63 & 5.14 \\
\hline 80 & 100 & 60 & 100 & 6.82 & 7.64 & 240.57 & 224.21 & 7.94 & 9.54 & 4.80 & 5.38 \\
\hline 60 & 100 & 60 & 100 & 5.56 & 6.17 & 204.25 & 190.57 & 7.63 & 9.07 & 4.14 & 4.59 \\
\hline 100 & 80 & 100 & 100 & 7.18 & 8.04 & 298.41 & 274.24 & 6.74 & 8.21 & 4.62 & 5.17 \\
\hline 80 & 80 & 100 & 100 & 7.05 & 7.83 & 256.86 & 235.28 & 7.69 & 9.32 & 4.77 & 5.30 \\
\hline 60 & 80 & 100 & 100 & 6.42 & 7.19 & 242.63 & 221.76 & 7.41 & 9.08 & 4.59 & 5.14 \\
\hline 100 & 80 & 80 & 100 & 7.20 & 7.76 & 261.39 & 242.83 & 7.72 & 8.95 & 4.95 & 5.34 \\
\hline 80 & 80 & 80 & 100 & 6.97 & 7.44 & 229.75 & 212.75 & 8.50 & 9.80 & 5.07 & 5.41 \\
\hline 60 & 80 & 80 & 100 & 6.13 & 6.60 & 216.51 & 201.14 & 7.93 & 9.19 & 4.72 & 5.09 \\
\hline 100 & 80 & 60 & 100 & 6.31 & 7.13 & 232.13 & 215.42 & 7.61 & 9.27 & 4.67 & 5.27 \\
\hline 80 & 80 & 60 & 100 & 6.19 & 7.18 & 218.46 & 203.60 & 7.94 & 9.88 & 4.86 & 5.64 \\
\hline 60 & 80 & 60 & 100 & 5.87 & 6.26 & 205.28 & 190.91 & 8.01 & 9.18 & 4.91 & 5.23 \\
\hline 100 & 60 & 100 & 100 & 4.42 & 4.91 & 256.52 & 235.74 & 4.83 & 5.83 & 3.14 & 3.49 \\
\hline 80 & 60 & 100 & 100 & 3.94 & 4.37 & 239.30 & 220.87 & 4.61 & 5.54 & 2.96 & 3.28 \\
\hline 60 & 60 & 100 & 100 & 3.39 & 3.70 & 214.95 & 196.89 & 4.42 & 5.26 & 2.71 & 2.95 \\
\hline 100 & 60 & 80 & 100 & 4.25 & 4.63 & 232.17 & 214.06 & 5.13 & 6.06 & 3.25 & 3.54 \\
\hline 80 & 60 & 80 & 100 & 3.73 & 3.99 & 209.73 & 192.74 & 4.98 & 5.80 & 3.04 & 3.25 \\
\hline 60 & 60 & 80 & 100 & 3.11 & 3.36 & 187.21 & 171.67 & 4.65 & 5.48 & 2.70 & 2.92 \\
\hline 100 & 60 & 60 & 100 & 3.78 & 4.04 & 203.85 & 187.13 & 5.19 & 6.05 & 3.14 & 3.35 \\
\hline 80 & 60 & 60 & 100 & 3.26 & 3.42 & 185.48 & 170.46 & 4.92 & 5.62 & 2.89 & 3.03 \\
\hline 60 & 60 & 60 & 100 & 2.46 & 2.61 & 171.32 & 157.79 & 4.02 & 4.63 & 2.34 & 2.49 \\
\hline \multirow{3}{*}{\multicolumn{2}{|c|}{ LSD 0.05}} & \multicolumn{2}{|c|}{ DI } & \multicolumn{2}{|c|}{0.01} & \multicolumn{2}{|c|}{0.99} & \multicolumn{2}{|c|}{0.04} & \multicolumn{2}{|c|}{0.01} \\
\hline & & \multicolumn{2}{|c|}{ IS } & \multicolumn{2}{|c|}{0.12} & \multicolumn{2}{|c|}{4.59} & \multicolumn{2}{|c|}{0.19} & \multicolumn{2}{|c|}{0.08} \\
\hline & & \multicolumn{2}{|c|}{ DI X IS } & \multicolumn{2}{|c|}{0.07} & \multicolumn{2}{|c|}{5.13} & \multicolumn{2}{|c|}{0.20} & \multicolumn{2}{|c|}{0.05} \\
\hline
\end{tabular}

Effect of DI under SDI and SSDI on WUE and IWUE

Data in Tables $12 \& 13$ show that the maximum values of water use efficiency (WUE) and irrigation water use efficiency (IWUE) for carrot roots were 9.86 and $5.61 \mathrm{~kg} \mathrm{~m}^{-3}, 9.88$ and $5.64 \mathrm{~kg}$ $\mathrm{m}^{-3}$ ) for seasons 2015-2016, respectively, under DI $(\mathrm{I}=80, \mathrm{D}=80, \mathrm{M}=60, \mathrm{~L}=100 \%)$ and SSDI treatment. While, the minimum values were 3.96 and $2.31 \mathrm{~kg} \mathrm{~m}^{-3}, 4.02$ and $2.34 \mathrm{~kg} \mathrm{~m}^{-3}$ for seasons 2015-2016, respectively, under DI $(\mathrm{I}=60, \mathrm{D}=60$, $\mathrm{M}=60, \mathrm{~L}=100 \%)$ and SDI treatment. Meanwhile, the values of WUE and IWUE under the DI $(I=80$, $\mathrm{D}=80, \mathrm{M}=60, \mathrm{~L}=100 \%$ ) and SSDI treatment for both seasons 2015-2016 increased significantly by approximately 50 and $28 \%$ compared with the control treatment (FI and SDI). These results may be because the deficit irrigation (DI) could be used 
to raise the crop yield to crop water consumption ratio where crops have growth stages in their development whereas they are tolerant to water stress. These results were similar to those reported by Ibragimov et al. (2007), Bekele \& Tilahun (2007), Geerts \& Raes (2009) and Quezada et al. (2011).

\section{Conclusion}

Results of the current study demonstrated beneficial effects, of DI during growth stages compared to FI under SDI and SSDI on the Ym, the studied quality parameters, seasonal ETa, WUE and IWUE for carrot roots. This study concluded that:

1- The quality parameters values, Ym and ETa for carrot roots decreased with increasing DI during growth stages except LAC TSC increased with increasing DI under SDI and SSDI for both seasons 2015 - 2016.

2- The maximum values of $\mathrm{Ym}$ were 8.38 and $8.56 \mathrm{Mg} \mathrm{fed}^{-1}$ for both seasons, respectively, under the FI ( $\mathrm{I}=100, \mathrm{D}=100, \mathrm{M}=100, \mathrm{~L}=100 \%)$ and SSDI treatment.

3- The minimum values of ETa were 154.00 and $157.79 \mathrm{~mm}$ for both seasons, respectively, under DI ( $I=60, \mathrm{D}=60, \mathrm{M}=60, \mathrm{~L}=100 \%)$ and SSDI treatment.

4- The values of WUE and IWUE under DI ( $I=80, D=80, M=60, L=100 \%$ ) and SSDI treatment for both seasons 2015-2016 increased significantly by approximately 50 and $28 \%$ compared with the control treatment (FI and SDI).

5- The initial and development growth stages for carrot roots were very sensitive to applicant DI for all treatments compared with midseason stage.

So, it is recommended to use DI $(\mathrm{I}=80, \mathrm{D}=80$, $\mathrm{M}=60, \mathrm{~L}=100 \%$ ) under SSDI treatment for Ym of carrot roots. This treatment save approximately $34 \%$ of the applied irrigation water although the reduction of Ym was approximately 4\% compared with the control treatment (FI and SDI). This means that the deficit irrigation technology during different growth stages can be recommended to apply on the other plants to find out sensitive and tolerant stages to apply it and therefore, save more irrigation water with less yield reduction.

\section{$\underline{\text { References }}$}

Agugo, B.A., Muoneke, C., Eno-Obongo, E. and Asiegbu, J.E. (2009) A Theoretical Estimate of Crop Evapotranspiration and Irrigation Water Requirements of Mungbean (Vigna radiata) in a Low Land Rain Forest Location of Southeastern Nigeria. Electronic Journal of Environmental, Agricultural and Food Chemistry. 8 (9),720-729.

Allen, R.G., Smith, M., Perrier, A. and Pereira, L.S. (1998) Crop Evapotranspiration, Guidelines for Computing Crop Water Requirements. FAO Irrigation and Drainage Paper No.56, FAO, Rome, Italy: 1-79.

Ayers, R.S. and Westcot, D.W. (1994) Water Quality for Agriculture, Irrigation and Drainage Paper No 29, FAO, Rome, Italy.

Bekele, S. and Tilahun, K. (2007) Regulated Deficit Irrigation Scheduling of Onion in a Semiarid Region of Ethiopia. Agricultural Water Management, 89, 148-152.

Doorenbos, J. and Pruitt, W.O. (1984) Crop Water requirements - Guidelines for predicting crop requirements. FAO Irrigation and Drainage Paper No.24, FAO, Rome, Italy: 45-90.

Geerts, S. and Raes, D. (2009) Deficit Irrigation as an On-Farm Strategy to Maximize Crop Water Productivity in, Dry Areas. Agricultural Water Management, 96, 1275-1284.

Howell, T. A. (2001) Enhancing water use efficiency in irrigated agriculture. Agronomy J. Abst. 93, 281 - 289.

Ibragimov, N., Evett, S.R., Esanbekov, Y., Kamilov, B.S., Mirzaev, L. and Lamers, J.P.A. (2007) Water Use Efficiency of Irrigated Cotton in Uzbekistan under Drip and Furrow Irrigation. Agricultural Water Management, 90, 112-120.

Kazemeini, S.A., Edalat, M. and Shekoofa, A. (2009) Interaction Effects of Deficit Irrigation and Row Spacing on Sunflower (Helianthus annuus L.) Growth, Seed Yield and Oil Yield. African Journal of Agricultural Research, 4 (11), 1165-1170.

Keller, J. and Karmeli, D. (1974) Trickle irrigation design parameters. ASAE, 17 (4), 678-684.

Klute, A. (1986) Methods of Soil Analysis, Part (1). Physical and Mineralogical Methods-Agronomy monograph No. 9 ( $2^{\text {nd }}$ edition). ASA and SSSA, Madison, WI, USA, $635-660$.

Leskovar, D. (2010) Scientists Complete Two-Year 
Study on Short-Day Onions. Science Daily 2010.

Michael, A. (1978) Irrigation and Theory Practice. Vikas Pub. House PVT LTD, New Delihi.

Nagaz, K., Masmoudi, M. and Ben Mechlia, N. (2012) Effect of Deficit Drip-Irrigation Scheduling Regimes with Saline Water on Pepper Yield, Water Productivity and Soil Salinity under Arid Conditions of Tunisia. Journal of Applied Horticulture, 14(1), $18-24$.

Owusu-Sekyere, J.D. and Andoh, J. (2011) Assessment of Deficit Irrigation on the Growth and Yield of Some Vegetable Crops; Department of Agricultural Engineering, University of Cape Coast, Ghana. Journal of Science and Technology, 35 (2), 143.

Page, A.L., Miller, R.H. and Keeney, D.R. (1982) Methods of Soil Analysis, part 2. Chemical and Microbiological Properties. Amer. Soc. of Agron, Madison, Wisconsin, USA.

Quezada, C., Fischer, S., Campos, J. and Ardiles, D. (2011) Water Requirements and Water Use Efficiency of Carrot under Drip Irrigation in a Haploxerand Soil. Journal of Soil Science and Plant Nutrition, 11 (1), $16-28$.
Sadgipour, O. (2008) Effect of Withholding Irrigation at Different Growth Stages on Yield and Yield Components on Mungbean (Vigna radiata $L$. Wilezek) Varieties. American-Eurasian Journal of Agricultural and Environmental Sciences, 4 (5), $590-594$.

Smith, M. (1992) CROPWAT A Computer Program for Irrigation Planning and Management and ETo calculation using Penman-Montieth method, FAO Irrigation and Drainage, Rome, Italy: 46: 112-140.

Snedecor, G.W. and Cochran, W.G. (1989) Statistical methods, $8^{\text {th }}$ ed., Iowa State Univ. Press, Iowa. USA: 476.

Webber, H., Madramootoo, C., Bourgault, M., Horst, M., Stulina, G. and Smith D. (2007) Plant-Soil Water Dynamics of Alternative Furrow and Regulated Deficit Irrigation for Two Legume Crops in the Fergana Valley, Uzbekistan. Water Saving in Mediterranean Agriculture and Future Research Needs. Bari: CIHEAM, 56 (1), 213-221.

(Received: $27 / 1 / 2017$ ) accepted: $6 / 4$ /2017) 
تأثير الري الناقص خلال مراحل النمو على كفاءة الأستهلاك المائى للجزرتحت ظروف الأسماعيلية النيز

على أحمد على عبد العزيز

قسم كيمياء وطبيعة الأر اضىى - مركز بحوث الصحر اء ـ القاهرة ـ مصر

مما لا شك فيه أن أستخدام تقنية الرى الناقص خلال مر احل النمو المختلفة تحت نظام الرى بالتنقيط تؤثربشكل

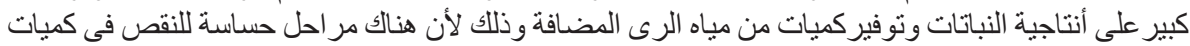

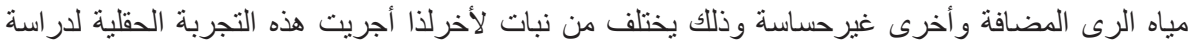

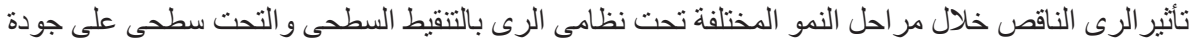

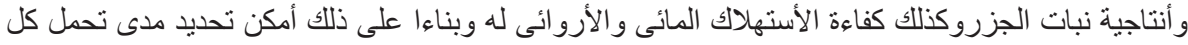

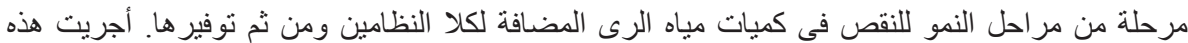

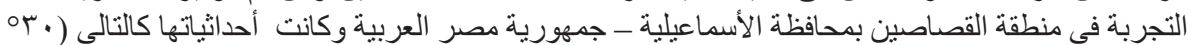

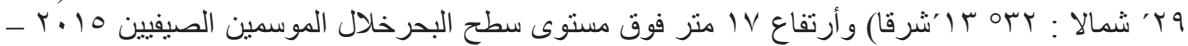

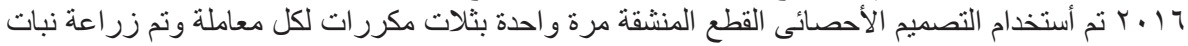

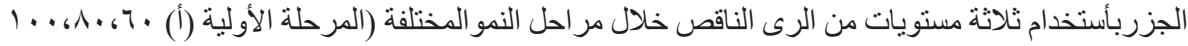

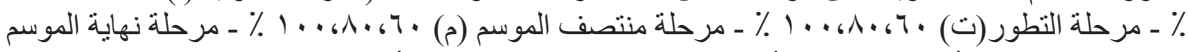

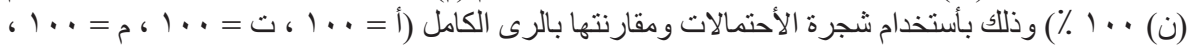
ن = . . (\%) تحت نظامى الرى بالتتقيط السطحى و التحت سطحى. وقد أوضحت النتائج الدتحصل عليها الأتى :

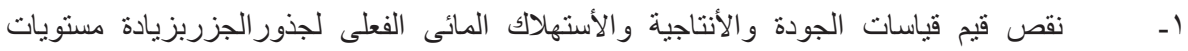

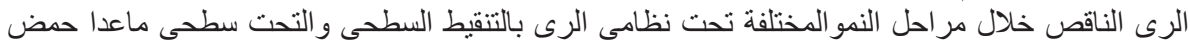

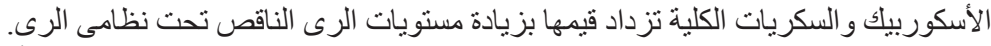

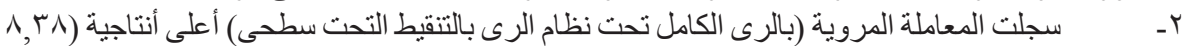

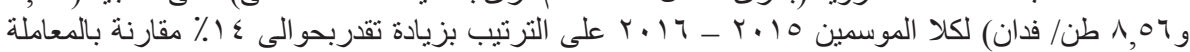

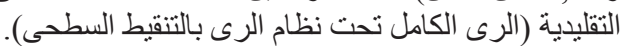

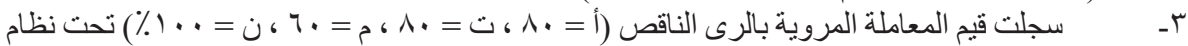

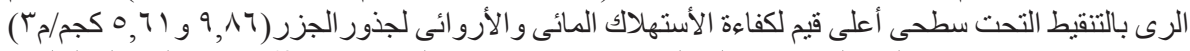

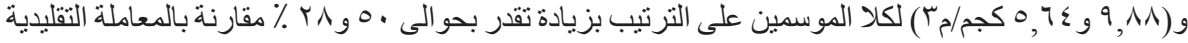
(الرى الكامل تحت نظام الرى بالتنقيط السطحى). عـ- - مرحلتى النموالأولية والتنور لجذور الجذرحساسة جدا للرى الناقص مقارنة بمرحلة منتصف

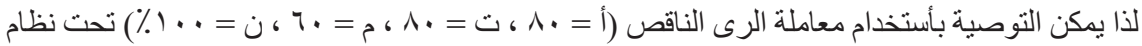

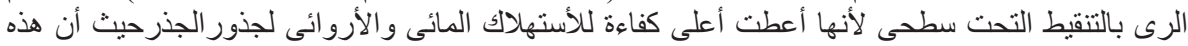

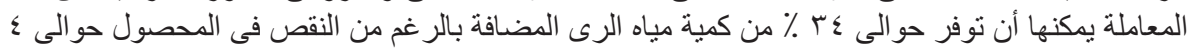

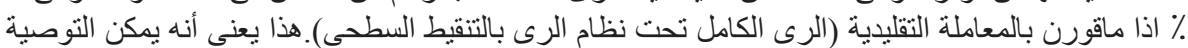

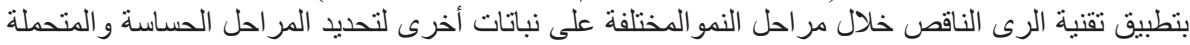
لنطبيقه وبالنالى توفير المزيد من كميات مياه الرى المضافة بأقل نقص في أنتاجية المحصول. 